TR Osborne, C Ramsenthaler, IJ Higginson. Palliative Care, Policy and Rehabilitation, King's College London, London, UK

Background Multiple myeloma is an incurable cancer, although recent treatment advances have improved expected survival from months to years in some cases. Increasing survival has elevated the importance of understanding and measuring patients' quality of life (QOL) in research and clinical settings. A number of QOL questionnaires exist for use in myeloma, yet there is a paucity of research to understand what issues are important to QOL from the patients' perspective.

This study explores the issues important to the QOL of people with myeloma, and how QOL is affected by the disease and its treatment. Implications for the design of QOL questionnaires are discussed.

Methods In-depth qualitative interviews with 20 myeloma patients to identify issues important to $\mathrm{QOL}$ and explore the impact of disease and treatment. Participants recruited from King's College Hospital and St. Christopher's Hospice, London. Purposive sampling by age, gender, performance status and disease stage. Thematic content analysis of interview transcripts was conducted.

Results Many participants started by discussing the importance of physical function and independence. There appeared to be a dominant role for participatory function in determining QOL - family life, leisure pursuits and other social activities were often identified as important. Physical symptoms such as pain or fatigue were frequently said to be affecting QOL, but often this was through their affect on social and participatory function. Sometimes symptoms were reported, but they did not affect QOL because functional and participatory aspects were maintained. Issues that prevented social engagement or participation were often therefore most deleterious to QOL - such as reduced mobility; susceptibility to infection; and fear of low-trauma fractures. Participants also often discussed the importance of quality, accessible healthcare - particularly good communication and receiving the right amount of information from professionals.

Conclusion These results support the broad consensus that quality of life is multidimensional, involving physical, psychological and social domains. The presence of symptoms per se did not necessarily impair QOL in this group. QOL questionnaires for use in myeloma should perhaps focus less on the presence, absence or severity of a symptom, but consider how much of a problem the symptom is in terms of its broader impact on function. This is particularly important when tools are applied in clinical practice, when the wider burden of symptoms is perhaps of greater interest. Tools for use in myeloma clinical practice should also consider items about the quality of healthcare - since this appears important to patients' $\mathrm{QOL}$.

\section{PS44 WHAT IS THE NATURE AND EXTENT OF ALCOHOL ADVERTISING ON FACEBOOK?}

doi:10.1136/jech-2012-201753.143

'B Page, ${ }^{2} \mathrm{~K}$ Cole. ' $C e n t r e$ for International Health and Development, UCL, London, UK; ${ }^{2}$ Department of Health Services Research and Policy, LSHTM, London, UK

Background There is a growing evidence base that alcohol advertising increases consumption, particularly amongst young people. Alcohol companies are increasingly using social media, such as Facebook, as a critical part of their marketing campaigns. The Advertising Standards Agency (ASA) states that alcohol advertising must not link alcohol with social success. However, by using a platform which is inherently social, alcohol companies can implicitly link their brand with these concepts. This study aimed to describe the nature and extent of alcohol advertising on Facebook.

Methods A quantitative descriptive analysis of UK Facebook brand pages of the highest volume sales brand for spirits, beer and cider (Smirnoff GB, Carling and Strongbow respectively) amongst the key Facebook user demographics (18-25 year olds). We collected all status updates for each brand page over a month. These appeared on the Newsfeed of those who Like the brand. The status updates were coded using concepts expressed in the ASA standards, the Committee of Advertising Practice (CAP) Code and the alcohol marketing literature. The frequencies of the identified categories were then calculated for each brand and for all three brands. The numbers of likes and comments of each status was also recorded.

Results 85 status updates were collected. The average number of status updates each day was 0.94 overall. The number of people receiving these updates at the time of writing is in total 881,398. The total number of likes and comments is 12,984 and 4,780 respectively. The most frequently occurring code was a reference to the Carling Cup. The codes used that refer to enhanced social or sexual success (which is in contravention of the ASA rules) were references to 'dating' and 'partying'. These occurred less often. All the brands encouraged user interaction by asking users a question (32 updates, $37.6 \%$ of all posts) and 'fill in the gap' statuses.

Conclusion This initial research suggests that the alcohol industry is using Facebook to engage with a large number of young consumers through frequent status updates. There is some evidence that the content on the pages is in contravention of ASA regulations. Further research needs to be conducted in this area in order to determine the effect that alcohol marketing via social media is having on levels of consumption of alcohol and initiation of consumption by young people in order to provide strong evidence for tightening ASA standards that reflect the advancement of alcohol advertising using Facebook

\section{PS45 ADDRESSING CHILD POVERTY - THE ROLE OF THE NHS WORKFORCE}

doi:10.1136/jech-2012-201753.144

JA Egan. Public Health Progammes, Glasgow Centre for Population Health, Glasgow, Scotland

Background Glasgow, like other UK cities, faces the challenge of addressing high child poverty levels - $52 \%$ of children, in some parts of the city, live in poverty. Within NHS Greater Glasgow and Clyde, the Healthier, Wealthier Children (HWC) project was set up to create referral pathways between the NHS and money advice services to support pregnant women and families at risk of child poverty. The 15 month project, launched in October 2010, was funded by the Scottish Government.

The evaluation, being undertaken by the Glasgow Centre for Population Health, will conclude in April 2012. This paper aims to describe the HWC evaluation findings with a focus on two key objectives: development of the NHS workforce role and mainstreaming child poverty action within this role.

Methods A mixed methods approach to the HWC evaluation included capturing and describing the changes in the NHS workforce roles through documentary analysis, an NHS workforce survey and outcomes from money advice services.

Results An HWC website was set up to provide staff materials, case studies and practical recommendations on child poverty work as an NHS workforce resource. Other ongoing work includes creating midwife/patient web-based scenarios to be used as a national resource.

Although not part of the existing NHS performance framework (i.e. HEAT targets), there is evidence that HWC work is being embedded. Most referrals to advice services were made by health visitors $(51 \%)$ and midwives $(29 \%)$. The majority of both workforces $(81 / 84 ; 96 \%)$ intend to continue referring post-HWC. Moreover, health visitors ranked clients' money worries as a priority above three national HEAT targets.

To date, 2,516 referrals were made to money advice services which resulted in uptake of $54 \%(n=1,347)$ among pregnant women 